\title{
Fiber Bragg grating based surface plasmon resonance sensor utilizing FDTD for alcohol detection applications
}

\begin{abstract}
A fiber Bragg grating (FBG) based surface plasmon resonance (SPR) sensor is investigated using 2-D finite difference time domain (FDTD) simulation. The sensitivity and accuracy of the sensor with and without gratings is observed as well as the effect of changing the grating distance on the resonant wavelength. The findings show that high sensitivity of above $500 \mathrm{~nm} / \mathrm{RIU}$ and a fourfold increase in signal to noise ratio (SNR) is obtained by introducing FBG to the basic fiber optic based SPR structure. Increasing the grating period results in a red shift in the resonant wavelength, thus enabling multiple sensor fabrication on a single fiber optic cable. Simulation of the FBG based SPR sensor to detect alcohol concentration from 0 to $100 \%$ in water is also demonstrated.
\end{abstract}

Keyword: Surface plasmon resonance; Fiber Bragg grating; Miniature sensors 